\title{
Combination of simvastatin and hydroxyapatite fiber induces bone augmentation
}

\author{
Shang $\mathrm{Gao}^{1^{*}}$, Makoto Shiota ${ }^{1}$, Masaki Fujii ${ }^{1}, \mathrm{Kang} \mathrm{Chen}^{1}$, Masahiro Shimogishi ${ }^{1}$, \\ Masashi Sato ${ }^{2}$, Shohei Kasugai ${ }^{1}$ \\ ${ }^{1}$ Department of Oral Implantology and Regenerative Dental Medicine, Tokyo Medical and Dental University, Tokyo, Japan; \\ *Corresponding Author: shang.irm@tmd.ac.jp \\ ${ }^{2}$ Department of Oral and Maxillofacial Surgery, Tokyo Medical and Dental University, Tokyo, Japan
}

Received 8 May 2013; revised 10 June 2013; accepted 13 July 2013

Copyright (C) 2013 Shang Gao et al. This is an open access article distributed under the Creative Commons Attribution License, which permits unrestricted use, distribution, and reproduction in any medium, provided the original work is properly cited.

\begin{abstract}
This study evaluated the capability of hydroxyapatite fiber (HAF) as a carrier and the bone formation by blending simvastatin. The mixture of HAF and simvastatin $(0.15,0.45,0.75 \mathrm{mg})$ was placed in $1 \mathrm{ml}$ of tris-buffer and the release of simvastatin from HAF was calculated per 24 hours for 10 days. Bilateral $5 \mathrm{~mm}$-diameter and 3 $\mathrm{mm}$-hight Teflon chambers were fixed on calvaria of adult Japanese white rabbits and filled with $40 \mathrm{mg}$ HAF which containing simvastatin $(0$, $0.15,0.45,0.75 \mathrm{mg}$ ). The animals were sacrificed at 4 and 8 weeks and calculated radiologically by Micro-CT. After dyeing by toluidine blue the samples were analyzed histologically. In all of the study groups approximately $25 \%$ of simvastatin was released until 10 days. The new bone volume ratio measured by Micro-CT of 4 and 8 weeks group was $(22.4 \%, 21.3 \%, 41.6 \%, 26.3 \%)$ and $(20.2 \%, 11.7 \%, 42.1 \%, 31.2 \%)$ in different doses respectively. The $0.45 \mathrm{mg}$ group showed significantly higher new bone volume ratio than 0 $\mathrm{mg}$ group and $0.15 \mathrm{mg}$ group. The histological measurement and observations also supported these results. In conclusion, the HAF could be used as a carrier for simvastatin. Combinations of HAF and simvastatin have the potentiality to stimulate new bone formation and approximately $0.45 \mathrm{mg}$ simvastatin in $\mathbf{4 0} \mathrm{mg}$ HAF is the optimal dose in rabbit chamber model.
\end{abstract}

Keywords: Biomaterial; Bone Substitutes; Bone Formation; Drug Delivery; Growth Factors

\section{INTRODUCTION}

With the increasing popularity of dental implants, the lack of sufficient amount of jaw bone has become a significant limitation. To overcome this constraint, researchers started focusing on bone regeneration in the context of dental implant surgery [1-4]. Over the past two decades, hydroxyapatite has proven to be an excellent bone substitute for its biocompatibility and space maintainability $[5,6]$. More recently, commercialization of hydroxyapatite bone substitute has been made possible, with most of the products being available as granular particles or blocks [7].

In clinical practice, however, these products have shown poor operational performance, for some clinicians opt to mix hydroxyapatite with venous blood or plateletrich plasma $[8,9]$ or use fiber-type hydroxyapatite. However, most of the initial attempts for obtaining pure hydroxyapatite fiber (HAF) have failed, as the adhesive or coating materials used drastically reduced its purity $[10,11]$. In recent years, pure HAF has been developed successfully, and its capacity to induce bone formation has been demonstrated [12,13]. Besides its effects on bone growth, HAF is also expected to serve as a carrier by holding substances within its three-dimensional structure and slowly releasing them-just like a drug-delivery system [14].

Growth factors, such as platelet-derived growth factor (PDGF), insulin-like growth factor (IGF), fibroblast growth factor (FGF), and bone morphogenetic protein (BMP)-2, have been reported to promote bone regeneration [15-18]. However, 2 issues limit their use in clinical practice: most of these growth factors are xenogeneic, and they are expensive. As an alternative, statins-widely known as competitive inhibitors of 3-hydroxy-3methylglutaryl coenzyme A (HMG-CoA) reductase that lower cholesterol levels [19] — have also been shown to increase BMP-2-induced bone formation [20,21]. Our previous study using simvastatin yielded similar results [22]. This effect appears to depend on the concentration 
of the drug, but high concentrations of simvastatin are unwanted because they provoke inflammation to the mandibular bone [23].

The aims of the present study were 1) to investigate the carrier properties of HAF by measuring the release of simvastatin from $\mathrm{HAF} /$ simvastatin compound materials; and 2) to measure bone regeneration induced by different blends of simvastatin combined with HAF.

\section{MATERIALS AND METHODS}

\subsection{Sample Preparation}

We used a previously described HAF compound (Fujii S., United State Patent No. 4,659,617) that was 5 - $15 \mu \mathrm{m}$ in diameter $[12,13]$. Energy dispersive X-ray analysis confirmed its purity; its three-dimensional structure is shown in Figure 1. Simvastatin (Ohara Pharmaceutical Co. Ltd., Koka, Shiga, Japan) was dissolved in ethanol. The solution was dropped onto HAF under sterile conditions, and then allowed to dry completely in a laminar flow hood for $24 \mathrm{~h}$. We prepared 4 groups of samples, each containing $40 \mathrm{mg} \mathrm{HAF}$ and $0 \mathrm{mg}, 0.15 \mathrm{mg}, 0.45 \mathrm{mg}$, and $0.75 \mathrm{mg}$ simvastatin, respectively.

\subsection{Measurement of Simvastatin Release}

The release of simvastatin from HAF was measured by using an ultraviolet - visible spectrophotometer (Nanodrop ND-1000; NanoDrop Technologies, Wilmington, DE, USA). The Nanodrop was calibrated using 8 standards of simvastatin solution at ambient temperature. The absorbance was measured at $238 \mathrm{~nm}$, and a standard curve for calculation of simvastatin concentrations was generated from the absorbance values. The samples were

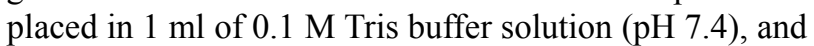
shaken on a Taitec Personal 11 Shaker (Taitec Corp., Tokyo, Japan) at $100 \mathrm{rpm}$ at ambient temperature. The amount of simvastatin released into the buffer was scored every $24 \mathrm{~h}$ for 10 days. The cumulative concentration was calculated by using the previously determined stan-

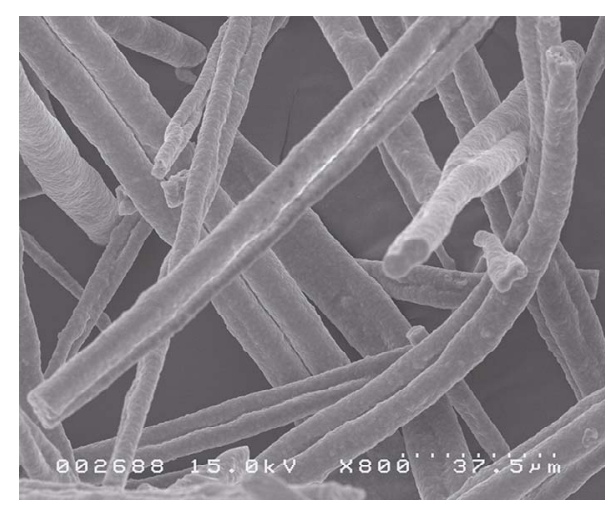

Figure 1. 3D-structure of HAF by SEM (magnification $\times 800$ ). dard curve.

\subsection{Surgical Procedures for the Rabbit Cranial Chamber Model}

The animal experimental protocol was approved by the Institutional Committee of Animal Care and Use at Tokyo Medical and Dental University. Twenty Japanese white rabbits weighing $2.5-3.0 \mathrm{~kg}$ were used. The animals were systemically anesthetized with an intramuscular injection of ketamine $(50 \mathrm{mg} / \mathrm{kg}$ Ketalar; Sankyo, Tokyo, Japan) and thiopental sodium ( $25 \mathrm{mg} / \mathrm{kg}$ Rabonal; Tanabe, Tokyo, Japan). The surgical area was shaved and prepared aseptically with povidone-iodine (Isodine Surgical Scrub; Meiji, Tokyo, Japan) for surgery. Before surgery, $1.8 \mathrm{ml}$ of a local anesthetic ( $2 \%$ xylocaine: epinephrine 1:80,000; Dentsply Sankin, Tokyo, Japan) was injected into the surgical site. Skin incision and dissection were carried out coronally, and periosteum incision and dissection were performed sagittally between the parietal and the frontal bone. After the periosteum was elevated, polytetrafluoroethylene chambers (hollow cylinders of $5.0 \mathrm{~mm}$ in diameter and $3.0 \mathrm{~mm}$ in height with an outer brim) were fixed with stainless steel screws (FKG Dentaire, Chaux-de-Fonds, Switzerland) to the parietal bone on the right and left sides. The chambers were filled with samples, which were selected randomly (Figure 2). The skin flaps were sutured with 4-0 nylon. During the observation period, all animals were given water and a standard feed ad libitum. Animals were sacrificed at 4 weeks or 8 weeks with a lethal dose of thiopental sodium. The entire cranial bone was harvested and fixed for 10 days in neutral $10 \%$ formalin.

\subsection{Micro-Computed Tomography (Micro-CT) Analysis}

The samples were scanned by a micro-CT scanner
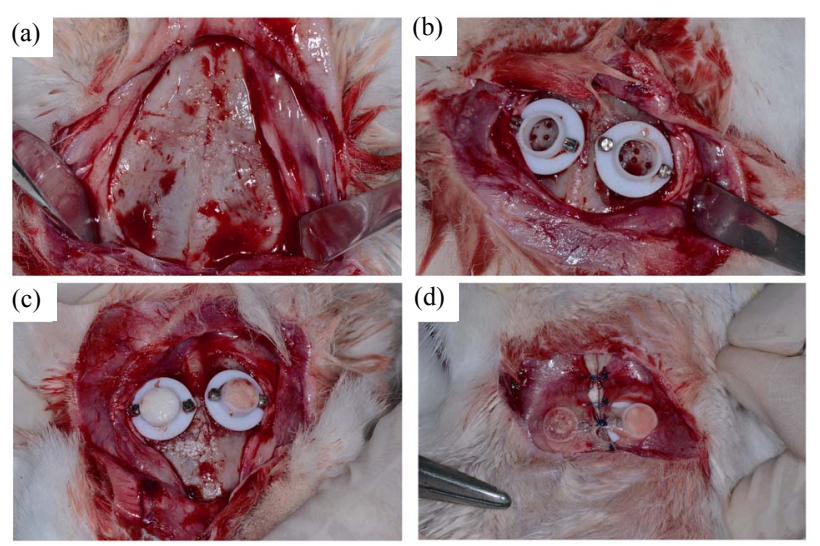

Figure 2. The animal surgical procedures. (a): Periosteum incision and elevating; (b): The chambers were fixed on both sides of rabbits; (c): The chambers were filled with samples; (d): Sutured with 4-0 nylon. 
(SMX-90CT; Shimadzu Science East Corporation, Tokyo, Japan) with a voxel size of $60 \mu \mathrm{m} /$ pixel, and quantified by using the Tri/3D-Bon software (Ratoc System Engineering Co. Ltd., Tokyo, Japan). The percentage of new bone volume occupying the total chamber volume was determined.

\subsection{Histological Processing}

After micro-CT analysis, the specimens were dehydrated in ascending grades of ethanol, following infiltration, and then embedded in methacrylate-based resin (Technovit 7200 VLC; Heraeus Kulzer, Wehrheim, Germany). The sections were cut and ground to a thickness of about $100 \mathrm{~m}$. The sections were finally stained with $0.1 \%$ toluidine blue. Histological observation was performed under a light microscope. The percentage of new bone in total chamber volume was measured with an image software (Photoshop CS6 extended, Adobe Systems complex, California, USA). The measurement was performed by a co-author.

\subsection{Statistical Analysis}

For statistical analysis, the data were tested by oneway analysis of variance (ANOVA). The data were further analyzed by Fisher's least significant difference test and Bonferroni multiple comparison test. All statistical analyses were performed using a commercial computer program (SPSS v. 11.5; SPSS Inc., Chicago, IL, USA). A value of $\mathrm{p}<0.05$ was considered to be statistically significant.

\section{RESULTS}

\subsection{Simvastatin Was Released from HAF in 2 Phases}

Approximately $10 \%$ of absorbed simvastatin was released after $24 \mathrm{~h}$. After this initial burst release, gradual and stable release of simvastatin was observed for 10 days (Figure 3). A similar release pattern was observed regardless of the initial concentration of simvastatin.

\subsection{Micro-CT Analysis}

Four weeks after surgical intervention, the percentage of new bone in the total chamber volume was $22.36 \% \pm 4.98 \%, 21.30 \% \pm 7.70 \%, 41.58 \% \pm 6.03 \%$, and $26.32 \% \pm 6.19 \%$ in the groups treated with different simvastatin/HAF ratios $(0 / 40 \mathrm{mg}, 0.15 / 40 \mathrm{mg}$, $0.45 / 40 \mathrm{mg}$, and $0.75 / 40 \mathrm{mg}$, respectively). Significant differences were recognized between the $0 / 40 \mathrm{mg}$ and the $0.45 / 40 \mathrm{mg}$ groups $(\mathrm{p}=0.047)$, and between the $0.15 / 40 \mathrm{mg}$ and the $0.45 / 40 \mathrm{mg}$ groups $(\mathrm{p}=0.037)$. After 8 weeks, the percentage of new bone was $20.22 \%$ $\pm 5.53 \%, 11.72 \% \pm 3.53 \%, 42.14 \% \pm 4.43 \%$, and
$31.22 \% \pm 8.58 \%$, respectively. Significant differences were recognized between the $0 / 40 \mathrm{mg}$ and the $0.45 / 40$ $\mathrm{mg}$ groups $(\mathrm{p}=0.17)$, between the $0.15 / 40 \mathrm{mg}$ and the $0.45 / 40 \mathrm{mg}$ groups $(\mathrm{p}=0.002)$, and between the $0.15 / 40 \mathrm{mg}$ and the $0.75 / 40 \mathrm{mg}$ groups $(\mathrm{p}=0.031)$ (Figure 4).

\subsection{Histology}

The main histological findings are presented in Figures 5-8. Four weeks after surgical intervention, the inner space of HAF-filled chambers was maintained clear in each group (Figure 5), i.e., there was no connective tissue invagination from the top of the chambers. However, some blood cells were observed. We also noted that the HAF fragment was oriented in different directions from sample to sample. The newly formed bone was found towards the bottom of the chamber, close to the host bone, in both control and simvastatin/HAF $0.15 / 40 \mathrm{mg}$ groups. On the other hand, in the $0.45 / 40 \mathrm{mg}$ group, the newly formed bone was found approximately at middle-height level between top of the chamber and host bone; in the $0.75 / 40 \mathrm{mg}$ group, the newly formed bone progressed up to approximately one-quarter of the total height of the chamber. The new bone connected with the host bone

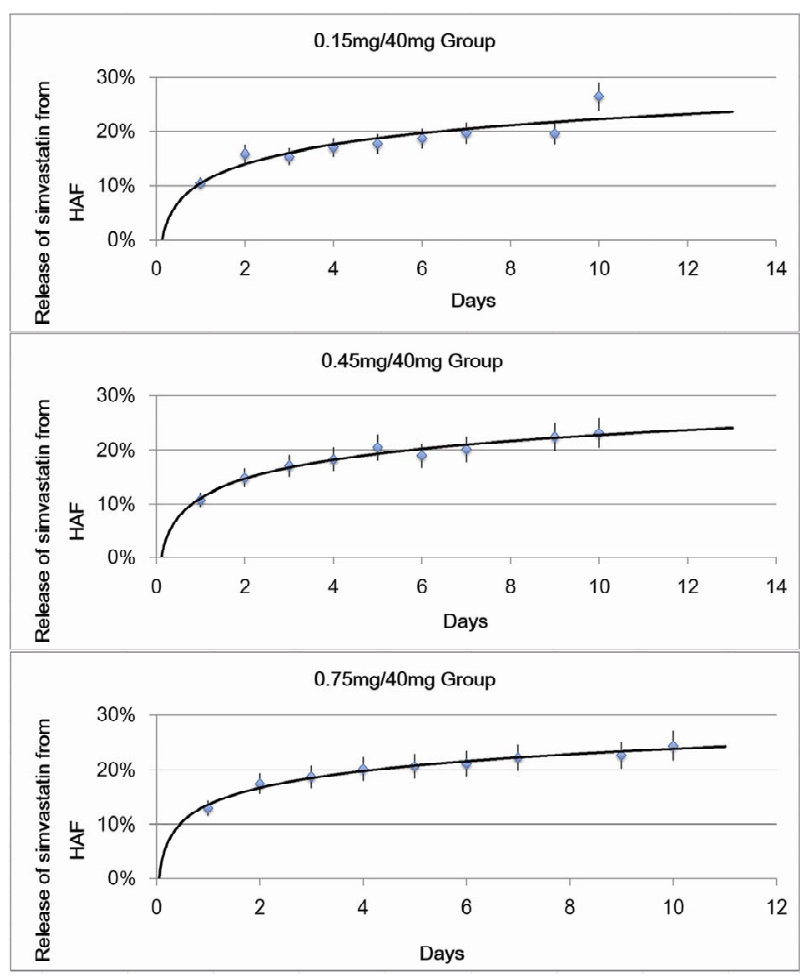

Figure 3. The release curve of simvastatin from HAF. Data were shown as mean $\pm \mathrm{SD}, \mathrm{n}=6$. After an approximately $10 \%$ initial burst release, stable release was observed for 10 days. Regardless of the difference concentrations of simvastatin, the release pattern was similar. 

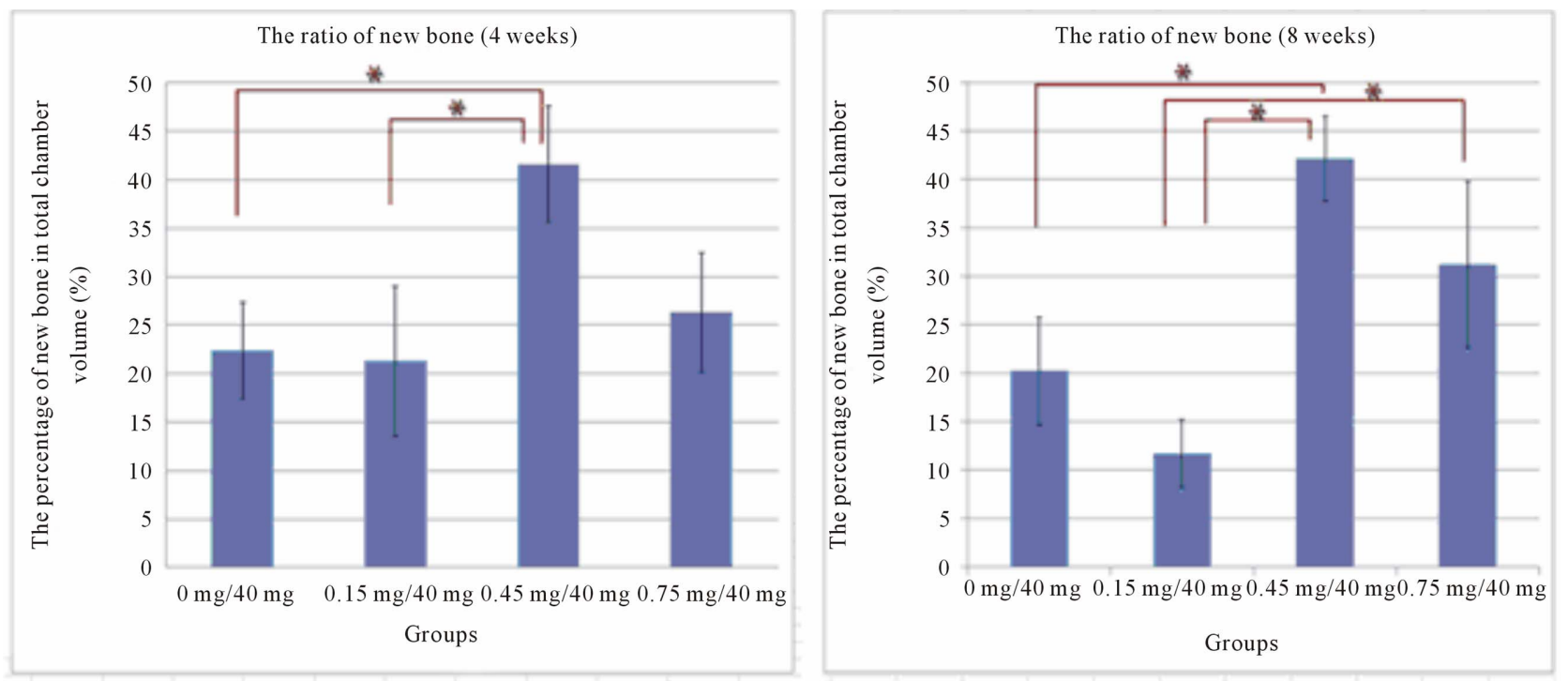

Figure 4. Micro-CT analysis, 4 weeks: The percentage of new bone in the total chamber volume was $22.36 \% \pm 4.98 \%, 21.30 \% \pm$ $7.70 \%, 41.58 \% \pm 6.03 \%$, and $26.32 \% \pm 6.19 \%$ in the groups treated with different simvastatin/HAF ratios. 8 weeks: The volume was $20.22 \% \pm 5.53 \%, 11.72 \% \pm 3.53 \%, 42.14 \% \pm 4.43 \%$, and $31.22 \% \pm 8.58 \%$, respectively $\left(\mathrm{n}=5,{ }^{*} \mathrm{p}<0.05\right)$.
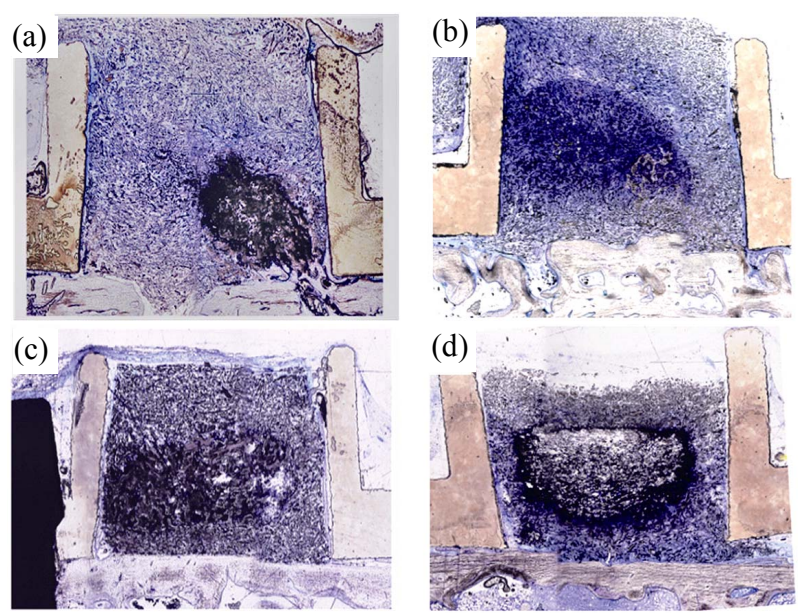

(d)

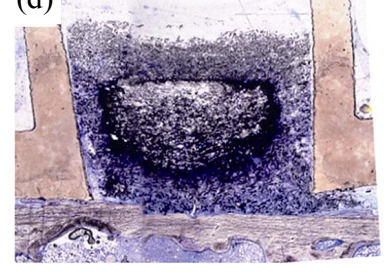

Figure 5. Four weeks after surgical intervention ((a): $0 / 40 \mathrm{mg}$ group (b): $0.15 / 40 \mathrm{mg}$ group (c): $0.45 / 40 \mathrm{mg}$ group (d): $0.75 / 40$ $\mathrm{mg}$ group). The diameter of chamber was $5.0 \mathrm{~mm}$. There was no connective tissue invagination from the top of the chambers. $0 / 40 \mathrm{mg}$ and $0.15 / 40 \mathrm{mg}$ groups: Towards the bottom of the chamber, the newly formed bone was found. $0.45 / 40 \mathrm{mg}$ group: The newly formed bone was found at middle-height level between top of the chamber and host bone. $0.75 / 40 \mathrm{mg}$ group: The new bone progressed up to one-quarter of the total chamber's height (Undecalcified samples, stained with toluidine blue).

and grew toward the HAF. It was possible to differentiate new bone from host bone based on differences in staining and on morphological appearance, i.e., new bone presented a "moth-eaten-like appearance" (Figure 6).

Eight weeks after surgical intervention, the inner space of the chambers was still clear and free of con-
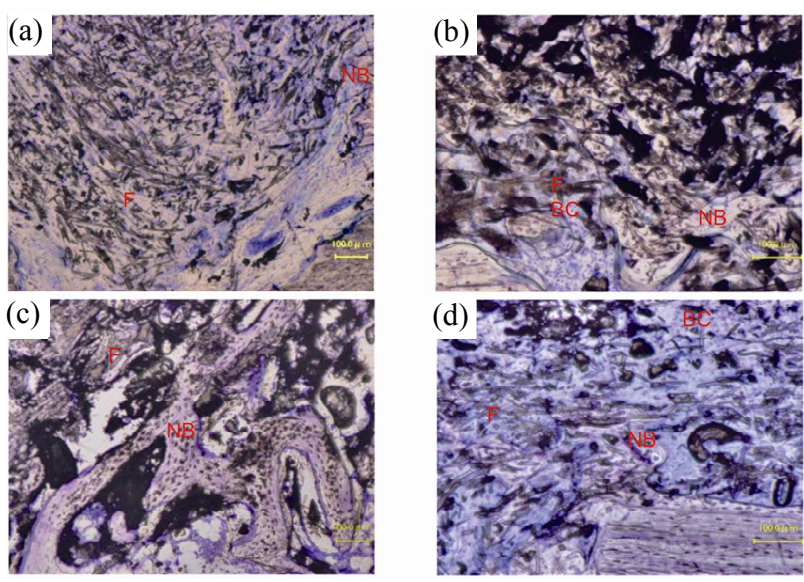

Figure 6. Four weeks after surgical intervention ((a): 0/40 mg group (b): $0.15 / 40 \mathrm{mg}$ group (c): $0.45 / 40 \mathrm{mg}$ group (d): $0.75 / 40$ $\mathrm{mg}$ group). The HAF fragment (F) was oriented in different directions. The staining and morphological appearance of new bone (NB) was different from the host bone. Some blood cells (BC) was observed between the NB and F. (Undecalcified samples, stained with toluidine blue).

nective tissue (Figure 7). However, the space between the HAF fragment-which was oriented in different directions from sample to sample - and the new bone was filled with blood cells and fat cells. In contrast to the samples dissected at 4 weeks after surgery, samples dissected at 8 weeks after surgery clearly showed the trabecular bone structure. Moreover, newly formed bone stained more similarly to host bone. The amount and relative position of new bone in the chambers were similar to those found at 4 weeks. The newly formed bone bridged with the host bone and showed a "moth- 
eaten-like appearance" (Figure 8). Inflammation was not observed at any of the 2 time points analyzed.

The results of histological measurement were similar to the micro-CT's (Figure 9). For four weeks, the percentage of new bone in the total chamber volume was $22.30 \% \pm 9.52 \%, 21.36 \% \pm 5.24 \%, 41.38 \% \pm 11.18 \%$, and $19.51 \% \pm 3.80 \%$ in the groups treated with different simvastatin/HAF ratios $(0 / 40 \mathrm{mg}, 0.15 / 40 \mathrm{mg}, 0.45 /$ $40 \mathrm{mg}$, and $0.75 / 40 \mathrm{mg}$, respectively). Significant differences were recognized between the $0 / 40 \mathrm{mg}$ and the $0.45 / 40 \mathrm{mg}$ groups $(\mathrm{p}=0.010)$, the $0.15 / 40 \mathrm{mg}$ and the $0.45 / 40 \mathrm{mg}$ groups $(\mathrm{p}=0.007)$, and between the 0.45 $\mathrm{mg} / 40 \mathrm{mg}$ and the $0.75 \mathrm{mg} / 40 \mathrm{mg}$ groups $(\mathrm{p}=0.003)$. After 8 weeks, the percentage of new bone was $22.45 \%$ $\pm 4.04 \%, 18.21 \% \pm 5.44 \%, 41.11 \% \pm 11.79 \%$, and $33.24 \% \pm 2.58 \%$, respectively. Significant differences were recognized between the $0 / 40 \mathrm{mg}$ and the $0.45 / 40$ $\mathrm{mg}$ groups $(\mathrm{p}=0.004)$, between the $0.15 / 40 \mathrm{mg}$ and the $0.45 / 40 \mathrm{mg}$ groups $(\mathrm{p}=0.001)$, and between the $0.15 / 40 \mathrm{mg}$ and the $0.75 / 40 \mathrm{mg}$ groups $(\mathrm{p}=0.020)$.

\section{DISCUSSION}

In this study, we confirmed the role of simvastatin in bone formation and regeneration. Simvastatin and other statins have been widely used for lowering serum levels of cholesterol in hypercholesterolemia, hyperlipidemia, and arteriosclerosis [19]. In recent years, several studies have shown that statins enhance BMP-2 and vascular endothelial growth factor (VEGF) gene expression in osteoblasts, suggesting their bone-promoting effect [21, 24,25]. Animal studies involving systemic or local administration of statins have also yielded positive results [23,26-28]. However, systemic administration of statins for bone formation requires a much more higher dose
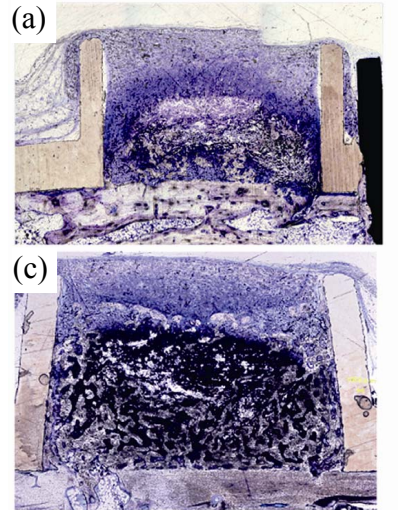

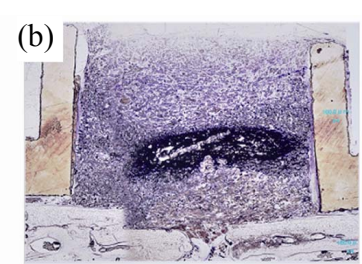

(d)

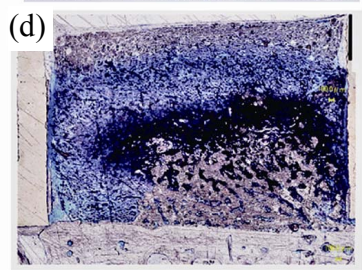

Figure 7. Eight weeks after surgical intervention ((a): 0/40 mg group (b): $0.15 / 40 \mathrm{mg}$ group (c): $0.45 / 40 \mathrm{mg}$ group (d): $0.75 / 40$ $\mathrm{mg}$ group). The diameter of chamber was $5.0 \mathrm{~mm}$. The inner space of chamber was clear and free of connective tissue. The new bone volume of each group was similar with 4 weeks but was stained more similarly to the host bone (Undecalcified samples, stained with toluidine blue).
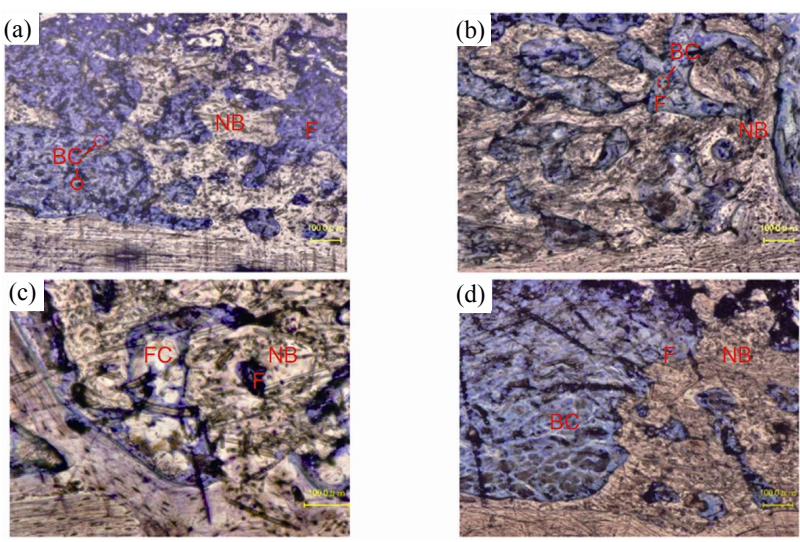

Figure 8. Eight weeks after surgical intervention ((a): 0/40 mg group (b): 0.15/40 mg group (c): 0.45/40 mg group (d): 0.75/40 $\mathrm{mg}$ group). The HAF fragment $(\mathrm{F})$ was oriented in different directions. Blood cells (BC) and fat cells (FC) were observed between the new bone (NB) and F. The NB clearly showed the trabecular structure, and bridged with the host bone showed a "moth-eaten-like appearance" (Undecalcified samples, stained with toluidine blue).

than for lowering cholesterol, and safety has not yet been clearly reported $[29,30]$. On the other hand, local administration can be performed with smaller doses, but it requires multiple or continuous injections [31]. In response to this drawback, local application of low-dose statin with a slow-release drug-delivery system has been suggested $[32,33]$.

In this study, we showed that HAF can serve as a substance carrier by demonstrating the stable release of simvastatin from $\mathrm{HAF} /$ simvastatin compound materials. This is in agreement with the report by Oda et al. [14], who suggested HAF as a potential component of a drug-delivery system based on its biocompatibility, biode-gradability, and cotton-like fibrous 3D structure. Importantly, the effects of HAF in bone formation have been demonstrated by using the rabbit cranium chamber model [12] and the post-extraction tooth socket model [13]. Kimura et al. [12] demonstrated that the combination of HAF with autogenous bone was effective in vertical bone augmentation, and Machida et al. [13] indicated that HAF could not only allow but also promote bone healing of the socket after tooth extraction. Therefore, we used simvastatin as growth-factor-like substance and HAF as scaffold in the present study. Here, an in vitro study showed that simvastatin is released gradually over time after an initial burst release from HAF. This release pattern was considered to be similar to that observed in several studies using other biomaterials, including alphatricalcium phosphate (TCP) [34], collagen sponge [35], electrospun fiber material [36], as the drug-delivery system. The bone-promoting effect of simvastatin correlated well with concentration [37]; thus, the initial burst release was thought to be a critical phase [34]. Addition- 

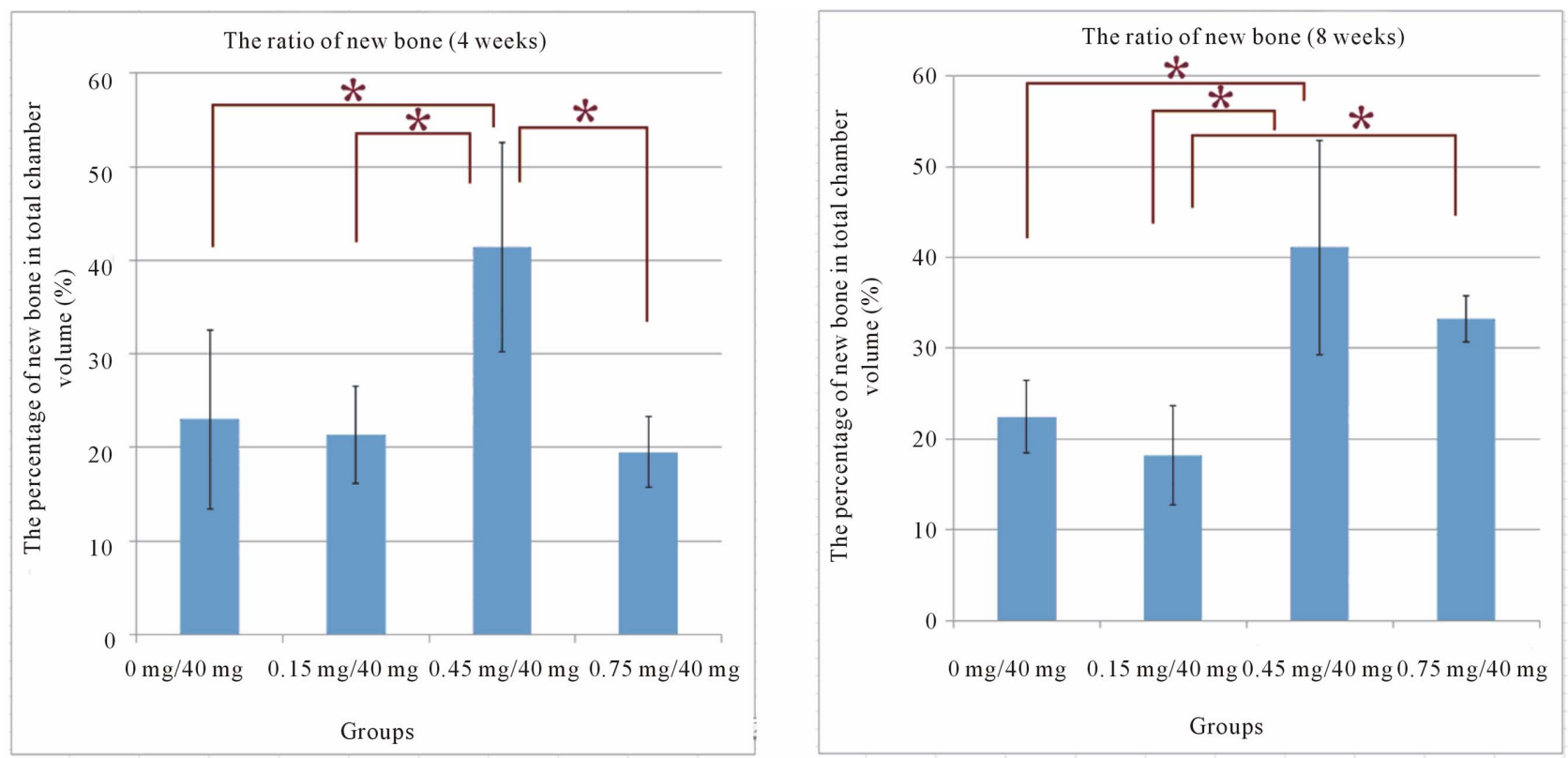

Figure 9. Histological analysis, 4 weeks: The percentage of new bone in the total chamber volume was $22.30 \% \pm 9.52 \%, 21.36 \% \pm$ $5.24 \%, 41.38 \% \pm 11.18 \%$, and $19.51 \% \pm 3.80 \%$ in the groups treated with different simvastatin/HAF ratios. 8 weeks: The volume was $22.45 \% \pm 4.04 \%, 18.21 \% \pm 5.44 \%, 41.11 \% \pm 11.79 \%$, and $33.24 \% \pm 2.58 \%$, respectively $\left(\mathrm{n}=5,{ }^{*} \mathrm{p}<0.05\right)$.

ally the release pattern always showed a constant curve regardless of concentration, indicating that the correlation coefficient of the release volume might be similar. Therefore, the release of simvastatin was considered to be adjusted by altering the amount of simvastatin added initially.

In the animal experiment, we used the rabbit cranial chamber model. Traditionally, the cranial bone defect model [38], tibia bone defect model [39], and post-extraction tooth socket model [40] were used to evaluate new bone formation in vivo. However, these models are not always rigorous enough to identify and evaluate the extent of new bone formation, since in these models bone can heal without pharmacological treatment [41]. Instead, the rabbit cranial chamber model offers a good model for new bone formation; in this model, the amount of new bone can be evaluated conveniently since the size of the chamber is constant. In this study, $40 \mathrm{mg}$ HAF were used. This dose had been demonstrated as the optimal dose for bone formation by Fujii et al. (2013) with using the same animal model.

To quantify the amount of newly formed bone, we used micro-CT analysis and histological measurement. Both showed the similar results, that suggested the validity of estimation method. We observed that $0.45 \mathrm{mg} \mathrm{sim}-$ vastatin induced significantly greater bone formation than lower amounts added to HAF at 4 weeks and 8 weeks after surgery, respectively. This strongly suggests that the bone-promoting effect of simvastatin is dosedependent, with $0.45 / 40 \mathrm{mg}$ simvastatin/HAF being the most effective combination. In previous studies, Nyan et al. [37] applied $0.1 \mathrm{mg}$ simvastatin with $14 \mathrm{mg}$ alphaTCP to rat calvarial defects, and Pradeep et al. [32] performed a randomized trial to treat chronic periodontitis with approximately $1.2 \%$ simvastatin solution. Both studies showed positive results with simvastatin concentrations that were similar to the most effective dose of our study $(0.45 / 40 \mathrm{mg})$.

Histological observations supported the above-mentioned findings. In addition, staining intensity and morphology of new bone at 8 weeks were more similar to host bone than at 4 weeks, which suggested the advanced maturation of new bone within 8 weeks. However, at an earlier time point, similar staining intensity and morphology of new bone were observed regardless of the different concentrations of simvastatin, which indicated that simvastatin could stimulate bone formation but not bone maturation. In agreement with our observations, Lee et al. [42] suggested that it was possible for simvastatin to stimulate bone formation but not bone maturation, and that much of the newly formed bone by simvastatin was resorbed in the long term.

Some researchers reported that a high dose of simvastatin could evoke inflammatory response in animal studies $[23,37]$, but no inflammation was found in the present study. This indicates that our highest dose $(0.75 \mathrm{mg})$ might not have been high enough to cause inflammatory reaction. Another curious effect of simvastatin is ectopic bone formation [14]. In our study model, in which the chamber was covered by periosteum, some mesenchymal cells could have had the chance to enter from the top of the chamber. However, there was no new bone formed at 
the top level of the chamber. Instead, newly formed bone connected with the host bone tightly.

In conclusion, this study revealed that HAF has the potential to be used as a carrier for simvastatin. Combinations of HAF and simvastatin stimulated new bone formation in a dose-dependent manner. Under our experimental conditions, we found $0.45 / 40 \mathrm{mg}$ simvastatin/ HAF to be the optimal combination dose for bone formation.

\section{CONCLUSION}

In conclusion, this study revealed that HAF has the potential to be used as a carrier for simvastatin. Combinations of HAF and simvastatin stimulated new bone formation in a dose-dependent manner. Under our experimental conditions, we found out that $0.45 / 40 \mathrm{mg}$ simvastatin/HAF is the optimal combination dose for bone formation.

\section{REFERENCES}

[1] Hermann, J.S. and Buser, D. (1996) Guided bone regeneration for dental implants. Current Opinion in Periodontology, 3, 168-77.

[2] Becker, W. and Becker, B.E. (1990) Guided tissue regeneration for implants placed into extraction sockets and for implant dehiscences: Surgical techniques and case report. The International Journal of Periodontics and Restorative Dentistry, 10, 376-91.

[3] Porter, J.R., Ruckh, T.T. and Popat, K.C. (2009) Bone tissue engineering: A review in bone biomimetics and drug delivery strategies. Biotechnology Progress, 25, 15391560 .

[4] Rogers, G.F. and Greene, A.K. (2012) Autogenous bone graft: Basic science and clinical implications. Journal of Craniofacial Surgery, 23, 323-327. doi:10.1097/SCS.0b013e318241dcba

[5] Bucholz, R.W., Carlton, A. and Holmes, R. (1989), Interporous hydroxyapatite as a bone graft substitute in tibial plateau fractures. Clinical Orthopaedics and Related Research, 240, 53-62.

[6] LeGeros, R.Z. (2002) Properties of osteoconductive biomaterials: Calcium phosphates. Clinical Orthopaedics and Related Research, 395, 81-98. doi:10.1097/00003086-200202000-00009

[7] Kunert-Keil, C., et al. (2009) Morphological evaluation of bone defect regeneration after treatment with two different forms of bone substitution materials on the basis of BONITmatrix. Canadian Journal of Physiology and Pharmacology, 60, 57-60.

[8] Grageda, E. (2004) Platelet-rich plasma and bone graft materials: A review and a standardized research protocol. Implant Dentistry, 13, 301-309.

[9] Faratzis, G., et al. (2012) Effect of autologous platelet-rich plasma in combination with a biphasic synthetic graft material on bone healing in critical-size cranial de- fects. Journal of Craniofacial Surgery, 23, 1318-1323. doi:10.1097/SCS.0b013e31825c76e5

[10] Dimitrievska, S., et al. (2008) Biocompatibility of novel polymer-apatite nanocomposite fibers. Journal of Biomedical Materials Research Part A, 84, 44-53. doi: $10.1002 / \mathrm{jbm} . \mathrm{a} .31338$

[11] Stanishevsky, A., et al. (2008) Hydroxyapatite nanoparticle loaded collagen fiber composites: Microarchitecture and nanoindentation study. Journal of Biomedical Materials Research Part A, 86, 873-882. doi:10.1002/jbm.a.31657

[12] Kimura, J., et al. (2012) Effect of hydroxyapatite fiber material with autogenous bone graft on vertical bone augmentation. Journal of Oral Tissue Engineering, 9, 136146.

[13] Machida, T., et al. (2010) Effect of hydroxyapatite fiber material on rat incisor socket healing. Journal of Oral Tissue Engineering, 7, 153-162.

[14] Oda, M., et al. (2009) Hydroxyapatite fiber material with BMP-2 gene induces ectopic bone formation. Journal of Biomedical Materials Research Part B, 90, 101-109.

[15] Hollinger, J.O., et al. (2008) Recombinant human platelet-derived growth factor: Biology and clinical applications. The Journal of Bone \& Joint Surgery, 90, 48-54. doi:10.2106/JBJS.G.01231

[16] Bernstein, A., Mayr, H.O. and Hube, R. (2010) Can bone healing in distraction osteogenesis be accelerated by local application of IGF-1 and TGF-beta1? Journal of Biomedical Materials Research Part B, 92, 215-225. doi:10.1002/jbm.b.31508

[17] Shimizu, E., et al. (2006) Fibroblast growth factor 2 and cyclic AMP synergistically regulate bone sialoprotein gene expression. Bone, 39, 42-52. doi:10.1016/j.bone.2005.12.011

[18] Visser, R., et al. (2009) The effect of an rhBMP-2 absorbable collagen sponge-targeted system on bone formation in vivo. Biomaterials, 30, 2032-2037. doi:10.1016/j.bone.2005.12.011

[19] Hunninghake, D., et al. (1998) Treating to meet NCEPrecommended LDL cholesterol concentrations with atorvastatin, fluvastatin, lovastatin, or simvastatin in patients with risk factors for coronary heart disease. The Journal of Family Practice, 47, 349-356.

[20] Mundy, G., et al. (1999) Stimulation of bone formation in vitro and in rodents by statins. Science, 286, 1946-1949. doi:10.1126/science.286.5446.1946

[21] Garrett, I.R., Gutierrez, G., and Mundy, G.R. (2001) Statins and bone formation. Current Pharmaceutical Design, 7, 715-736. doi: 10.2174/1381612013397762.

[22] Nyan, M., et al. (2007) Bone formation with the combination of simvastatin and calcium sulfate in critical-sized rat calvarial defect. Journal of Pharmacological Sciences, 104, 384-386. doi:10.1254/jphs.SC0070184

[23] Stein, D., et al. (2005) Local simvastatin effects on mandibular bone growth and inflammation. Journal of Periodontology, 76, 1861-1870. doi:10.1902/jop.2005.76.11.1861

[24] Ohnaka, K., et al. (2001) Pitavastatin enhanced BMP-2 
and osteocalcin expression by inhibition of Rho-associated kinase in human osteoblasts. Biochemical and Biophysical Research Communications, 287, 337-342. doi:10.1006/bbrc.2001.5597.

[25] Maeda, T., Kawane T. and Horiuchi, N. (2003) Statins augment vascular endothelial growth factor expression in osteoblastic cells via inhibition of protein prenylation. Endocrinology, 144, 681-692. doi:10.1210/en.2002-220682

[26] Junqueira, J.C., et al. (2002) Effects of simvastatin on bone regeneration in the mandibles of ovariectomized rats and on blood cholesterol levels. Oral Science International, 44, 117-124. doi:10.2334/josnusd.44.117

[27] Pytlik, M., et al. (2003) Effects of simvastatin on the development of osteopenia caused by ovariectomy in rats. Polish Journal of Pharmacology, 55, 63-71.

[28] Morris, M.S., et al. (2008) Injectable simvastatin in periodontal defects and alveolar ridges: Pilot studies. Journal of Periodontology, 79, 1465-1473. doi:10.1902/jop.2008.070659

[29] Todd, P.A. and Goa, K.L. (1990) Simvastatin. A review of its pharmacological properties and therapeutic potential in hypercholesterolaemia. Drugs, 40, 583-607. doi:10.2165/00003495-199040040-00007

[30] Tikiz, C., et al. (2005) Effects of simvastatin on bone mineral density and remodeling parameters in postmenopausal osteopenic subjects: 1-year follow-up study. Clinical Rheumatology, 24, 447-52. doi:10.1007/s10067-004-1053-x

[31] Skoglund, B. and Aspenberg, P. (2007) Locally applied Simvastatin improves fracture healing in mice. $B M C$ Musculoskeletal Disorders, 8, 98. doi:10.1186/1471-2474-8-98

[32] Pradeep, A.R. and Thorat, M.S. (2010) Clinical effect of subgingivally delivered simvastatin in the treatment of patients with chronic periodontitis: A randomized clinical trial. Journal of Periodontology, 81, 214-222. doi:10.1902/jop.2009.090429

[33] Tanigo, T., Takaoka R. and Tabata, Y. (2010) Sustained release of water-insoluble simvastatin from biodegradable hydrogel augments bone regeneration. Journal of Con- trolled Release, 143, 201-206. doi:10.1016/j.jconrel.2009.12.027

[34] Nyan, M., et al. (2010) Molecular and tissue responses in the healing of rat calvarial defects after local application of simvastatin combined with alpha tricalcium phosphate. Journal of Biomedical Materials Research Part B: Applied Biomaterials, 93, 65-73.

[35] Monjo, M., et al. (2010) In vivo performance of absorbable collagen sponges with rosuvastatin in criticalsize cortical bone defects. Acta Biomaterialia, 6, 1405-1412. doi:10.1016/j.actbio.2009.09.027

[36] Wadagaki, R., et al. (2011) Osteogenic induction of bone marrow-derived stromal cells on simvastatin-releasing, biodegradable, nano- to microscale fiber scaffolds. Annals of Biomedical Engineering, 39, 1872-1881. doi:10.1007/s10439-011-0327-0

[37] Nyan, M., et al. (2009) Effects of the combination with alpha-tricalcium phosphate and simvastatin on bone regeneration. Clinical Oral Implants Research, 20, 280-287. doi:10.1111/j.1600-0501.2008.01639.x

[38] Sakai, K., et al. (2011) Effects on bone regeneration when collagen model polypeptides are combined with various sizes of alpha-tricalcium phosphate particles. Dental Materials, 2011. doi:10.4012/dmj.2011-126

[39] Batista, M.A., et al. (2011) Comparison between the effects of platelet-rich plasma and bone marrow concentrate on defect consolidation in the rabbit tibia. Clinics (Sao Paulo), 66, 1787-92.

[40] Hong, H.H., et al. (2012) The potential effects of cholecalciferol on bone regeneration in dogs. Clinical Oral Implants Research, 23, 1187-1192. doi:10.1111/j.1600-0501.2011.02284.x

[41] Schmitz, J.P. and Hollinger, J.O. (1986) The critical size defect as an experimental model for craniomandibulofacial nonunions. Clinical Orthopaedics and Related Research, 205, 299-308.

[42] Lee, Y., et al. (2008) The effect of local simvastatin delivery strategies on mandibular bone formation in vivo. Biomaterials, 29, 1940-1949. doi:10.1016/j.biomaterials.2007.12.045 